\title{
Evidence for thymectomy in myasthenia gravis: Getting stronger?
}

\author{
Marc de Perrot, MD, ${ }^{\mathrm{a}}$ and Karen McRae, $\mathrm{MD}^{\mathrm{b}}$
}

\begin{abstract}
Thymectomy is part of the therapeutic armamentarium for myasthenia gravis (MG). During the past 80 years, multiple observational studies have shown that thymectomy can potentially fasten stabilization of the disease, reduce the need for corticosteroids, and in some patients lead to complete remission. The benefit from thymectomy in MG is supported by propensity score analysis. A randomized control trial looking at the role of thymectomy in nonthymomatous MG patients was recently completed, but the results are not yet available. The approach and extent of thymectomy remain a topic of intense discussion, particularly with the development of minimally invasive surgery. Although the presence of extracapsular thymic tissue is frequent and well described, the accessibility of these ectopic thymic foci as well as their function and impact on outcome after surgery have been unclear, leading to divergent views between proponents of a maximal cervicomediastinal thymectomy with en bloc resection of all fatty tissue between the thyroid grand and the diaphragm and those of a less extensive approach. In the future, better definition of the type of thymectomy will be important, particularly if prospective studies and randomized trials are performed to compare different surgical approaches. One possibility would be to reserve the term "extended thymectomy" to resection of the thymus with the anterior mediastinal fat between both pleura, the pericardium and diaphragm. More extensive surgery should be specified when it encompasses sites such as the right and left pericardiophrenic angles, the aortopulmonary window, the aortocaval groove and retroinnominate space, and the perithyroid area. (J Thorac Cardiovasc Surg 2017;154:314-6)
\end{abstract}

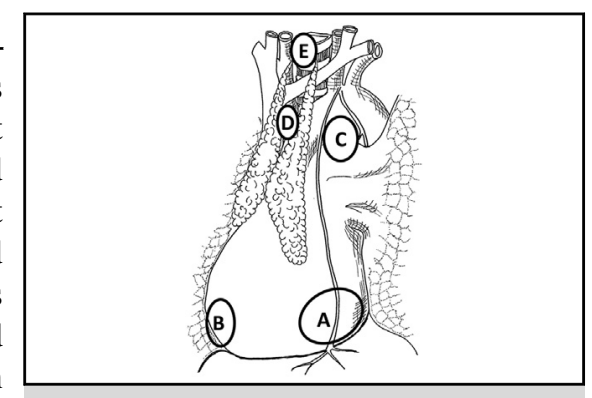

Thymectomy for myasthenia gravis can include specific additional sites in the mediastinum.

\section{Central Message}

Patient subtypes and descriptions of operations performed could prove important in future trials of thymectomy for myasthenia gravis.

\section{Perspective}

The term extended thymectomy should be defined as resection of the thymus with the anterior mediastinal fat between both pleura, the pericardium, and the diaphragm. More extensive surgery can encompass sites such as the right and left pericardiophrenic angles, the aortopulmonary window, the aortocaval groove and retroinnominate space, and the perithyroid area.
Myasthenia gravis (MG) is characterized by autoantibodies against components of the neuromuscular junction. The clinical presentation and pathogenic mechanisms are heterogeneous, with a first peak in incidence in the second and third decades of life affecting mostly women and a second peak in the sixth and seventh decades affecting both sexes. Thymic hyperplasia is most frequently encountered in young patients and thymoma in elderly patients.

\footnotetext{
From the ${ }^{\mathrm{a}}$ Division of Thoracic Surgery and ${ }^{\mathrm{b}}$ Department of Anesthesia and Pain Management, Toronto General Hospital, University Health Network, University of Toronto, Toronto, Ontario, Canada.

Received for publication Jan 3, 2016; accepted for publication Jan 5, 2016; available ahead of print Feb 12, 2016.

Address for reprints: Marc de Perrot, MD, Division of Thoracic Surgery, Toronto General Hospital, 9N-961, 200 Elizabeth St, Toronto, Ontario M5G 2C4, Canada (E-mail: marc.deperrot@uhn.ca).

$0022-5223 / \$ 36.00$

Copyright $(c) 2016$ by The American Association for Thoracic Surgery

http://dx.doi.org/10.1016/j.jtcvs.2016.01.006
}

The first step in understanding the mechanisms of MG occurred in the early 1930s, when the similarity between curare poisoning and MG was recognized and cholinesterase inhibitors were introduced as treatment. Only in 1973 was MG recognized as an autoimmune disorder, which led to the introduction of corticosteroids and other immunosuppressive drugs. These developments have been associated with remarkable progress, as demonstrated by a dramatic reduction in the mortality of patients with MG and improvement in their quality of life.

The understanding of the mechanisms of MG has been refined in the past 15 years with the characterization of several new autoimmune antibodies affecting the neuromuscular junction: muscle-specific kinase antibodies, lowdensity lipoprotein receptor-related protein 4 antibodies, agrin antibodies, titin antibodies, and ryanodine receptor antibodies. These observations have led to the development of a subgroup classification of MG that is based on the types 


\section{Abbreviation and Acronym \\ $\mathrm{MG}=$ myasthenia gravis}

of autoantibodies, with implications for diagnosis, therapy, and prognosis. ${ }^{2}$ MG may be classified as follows: (1) the most prevalent MG subtype, with autoantibodies against the muscle-type acetylcholine receptor; (2) MG with antibodies against muscle-specific kinase; (3) MG with antibodies against the agrin-receptor low-density lipoprotein receptor-related protein, and (4) MG occurring without a known target autoantigen ("triple seronegative $M G$ "). The classification of muscle-type acetylcholine receptor antibody-related MG is further divided into 3 subgroups according to the age of the patient at the onset of symptoms and the presence of thymoma as follows: (1) early onset (patient younger than 50 years at presentation), (2) late onset (patient older than 50 years at presentation), and (3) thymoma (regardless of age).

The concept of thymectomy for patients with MG was introduced by Alfred Blalock in 1936. ${ }^{3}$ After his initial reports, thymectomy progressively gained widespread acceptance in the treatment of $\mathrm{MG}$ to achieve more rapid stabilization of the disease, a reduction in the need for corticosteroids, and in some cases complete remission. The benefit from thymectomy, however, has so far not been confirmed in a randomized trial. A multicenter, singleblind, randomized study comparing thymectomy with no thymectomy in patients with nonthymomatous MG with a predefined prednisone protocol was recently completed, and the results should be available in the near future. This trial enrolled 200 patients and involved 70 centers in 22 countries, highlighting the notorious difficulty of randomly allocating potential surgical candidates to a nonsurgical arm. ${ }^{4}$

Currently, thymectomy is commonly considered for patients with early-onset MG with muscle-type acetylcholine receptor autoantibodies. Benefit in this group of patients was recently confirmed by a propensity score analysis that demonstrated that thymectomy was associated with a higher probability of remission than was seen among patients who did not undergo surgery after controlling for age, sex, time to diagnosis, severity of symptoms at diagnosis, and the use of immunosuppressive therapy. ${ }^{5}$ With the exception of the subgroup of patients with thymoma requiring a thymectomy for oncologic reasons, the role of thymectomy in other subgroups of patients remains more controversial. Recent development in antibody testing may help to refine the indications for surgery in some of these patients, because increasing evidence suggests that thymectomy is not beneficial to patients with MG related to muscle-specific kinase antibodies. ${ }^{2}$
The approach and extent of thymectomy remain a topic of intense discussion, particularly since the development of minimally invasive surgery. Although the presence of extracapsular thymic tissue is frequent and well described, the accessibility of these ectopic thymic foci as well as their function and impact on outcome have been unclear, leading to divergent views between proponents of a maximal cervicomediastinal thymectomy, with en bloc resection of all fatty tissue between the thyroid grand and the diaphragm, and those who favor a minimally invasive approach.

A number of recent studies and observations do potentially mitigate the potential benefit of a maximal approach. (1) Ectopic thymic foci are usually isolated to 1 or 2 sites and are most frequently found in the anterior mediastinal fat. Other sites are often found along the phrenic and recurrent nerves or in an area not accessible to surgery. ${ }^{6,7}$ (2) Maximal thymectomy with resection of all potential sites of ectopic thymic foci in the neck and mediastinum is associated with a small but real risk of phrenic and recurrent nerve injury. ${ }^{8}$ (3) The presence of ectopic thymic foci is potentially one of the strongest predictors of poor outcome, even after maximal thymectomy. ${ }^{9,10}$ (4) Finally, ectopic thymic foci are found more frequently in patients with atrophic thymus than in patients with hypertrophic thymus, thus possibly indicating an increased risk-benefit ratio of a maximal approach in the latter group of patients. ${ }^{11}$

These findings suggest that resection of all active ectopic thymic tissue can be difficult and that the benefit may be limited. Considering that ectopic thymic foci are most frequently located in the anterior mediastinum and that this area is readily accessible to surgery, an extended thymectomy involving the fat located between the pericardium, both pleura, and the diaphragm appears to be a very good option. Masaoka and colleagues ${ }^{12}$ reported a complete remission rate of $46 \%$ at 5 years with this approach in patients with nonthymomatous MG. These results are better than in patients undergoing a less extensive thymectomy and are very similar to the results achieved with a maximal thymectomy with resection of tissue in the pericardiophrenic angles, in the aortopulmonary window, in the aortocaval groove, and along the thyroid gland. ${ }^{13-15}$ Extending the resection to areas other than the anterior mediastinal fat should therefore continue to be investigated, because it may potentially provide benefit for some subgroups of patients. Currently, however, there is limited evidence to suggest that such extension should be done routinely.

An extended thymectomy involving the anterior mediastinal fat can be performed by an open approach through a sternotomy or by a minimally invasive approach through a cervicotomy, transcervical-subxiphoid approach or unilateral or bilateral video-assisted thoracoscopic surgery. More recently, robotic thymectomy has also been used with success. The results from all the minimally invasive approaches have been relatively similar as long as an extended complete 


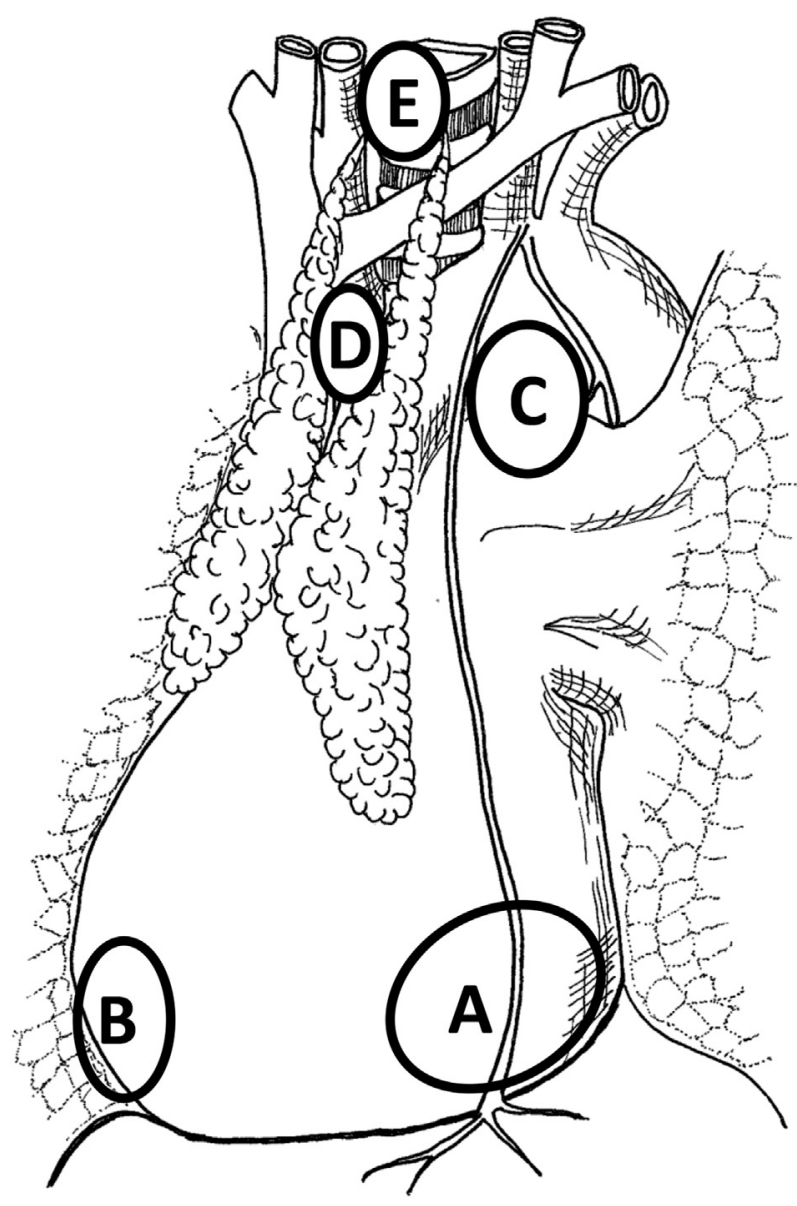

FIGURE 1. Extended thymectomy should encompass resection of the thymus with the anterior mediastinal fat between both pleura, the pericardium, and the diaphragm. More extensive surgery could include sites such as the right and left pericardiophrenic angles $(A$ and $B)$, the aortopulmonary window $(C)$, the aortocaval groove and retroinnominate space $(D)$, and the perithyroid area $(E)$.

thymectomy with at least resection of the anterior mediastinal fat was performed, and it is ultimately the surgeon's preference and comfort that should dictate the type of approach.

In the future, better definition of the type of thymectomy will be important, particularly if prospective studies and randomized trials are performed to compare different surgical approaches. One possibility would be to reserve the term extended thymectomy for resection of the thymus with the anterior mediastinal fat between both pleura, the pericardium, and the diaphragm. More extensive surgery could be specified to encompass sites such as the right and left pericardiophrenic angles, the aortopulmonary window, the aortocaval groove and retroinnominate space, and the perithyroid area (Figure 1).
In conclusion, the past 15 years have been associated with major changes in our understanding of MG. These developments will undoubtedly lead toward better care for these patients and help to refine the indications for thymectomy, targeting the most appropriate group of patients. A randomized trial of patients requiring steroid therapy for MG should soon provide additional information on the role of thymectomy in this patient population and will hopefully lead to further surgical trials to determine the best approach.

\section{Conflict of Interest Statement}

Authors have nothing to disclose with regard to commercial support.

\section{References}

1. Sieb JP. Myasthenia gravis: an update for the clinician. Clin Exp Immunol. 2014; 175:408-18.

2. Gilhus NE, Verschuuren JJ. Myasthenia gravis: subgroup classification and therapeutic strategies. Lancet Neurol. 2015;14:1023-36.

3. Blalock A. Thymectomy in the treatment of myasthenia gravis: report of 20 cases. J Thorac Surg. 1944;13:316-39.

4. Newsom-Davis J, Cutter G, Wolfe GI, Kaminski HJ, Jaretzki A III, Minisman G, et al. Status of the thymectomy trial for nonthymomatous myasthenia gravis patients receiving prednisone. Ann N Y Acad Sci. 2008;1132:344-7.

5. Barnett C, Katzberg HD, Keshavjee S, Bril V. Thymectomy for nonthymomatous myasthenia gravis: a propensity score matched study. Orphanet J Rare Dis. 2014;9:214.

6. Klimek-Piotrowska W, Mizia E, Kuzdzal J, Lazar A, Lis M, Pankowski J. Ectopic thymic tissue in the mediastinum: limitations for the operative treatment of myasthenia gravis. Eur J Cardiothorac Surg. 2012;42:61-5.

7. Fukai I, Funato Y, Mizuno T, Hashimoto T, Masaoka A. Distribution of thymic tissue in the mediastinal adipose tissue. J Thorac Cardiovasc Surg. 1991;101: 1099-102.

8. Bulkley GB, Bass KN, Stephenson GR, Diener-West M, George S, Reilly PA, et al. Extended cervicomediastinal thymectomy in the integrated management of myasthenia gravis. Ann Surg. 1997;226:324-34; discussion 334-5.

9. Ambrogi V, Mineo TC. Active ectopic thymus predicts poor outcome after thymectomy in class III myasthenia gravis. J Thorac Cardiovasc Surg. 2012;143: 601-6.

10. Ozdemir N, Kara M, Dikmen E, Nadir A, Akal M, Yucemen N, et al. Predictors of clinical outcome following extended thymectomy in myasthenia gravis. Eur J Cardiothorac Surg. 2003;23:233-7.

11. Chen Z, Luo H, Peng Y, Cai L, Zhang J, Su C, et al. Comparative clinical features and immune responses after extended thymectomy for myasthenia gravis in patients with atrophic versus hyperplastic thymus. Ann Thorac Surg. 2011;91: 212-8.

12. Masaoka A, Yamakawa Y, Niwa H, Fukai I, Kondo S, Kobayashi M, et al. Extended thymectomy for myasthenia gravis patients: a 20-year review. Ann Thorac Surg. 1996;62:853-9.

13. Masaoka A, Monden Y. Comparison of the results of transsternal simple, transcervical simple, and extended thymectomy. Ann N Y Acad Sci. 1981;377:755-65.

14. Jaretzki A III, Penn AS, Younger DS, Wolff M, Olarte MR, Lovelace RE, et al. "Maximal" thymectomy for myasthenia gravis: results. J Thorac Cardiovasc Surg. 1988;95:747-57.

15. Zielinski M, Hauer L, Hauer J, Pankowski J, Nabialek T, Szlubowski A. Comparison of complete remission rates after 5 year follow-up of three different techniques of thymectomy for myasthenia gravis. Eur J Cardiothorac Surg. 2010; 37:1137-43.

Key Words: mediastinum, myasthenia gravis, thymectomy 